\title{
On bounding the difference of the maximum degree and the clique number
}

\author{
Oliver Schaudt • Vera Weil
}

the date of receipt and acceptance should be inserted later

\begin{abstract}
For every $k \in \mathbb{N}_{0}$, we consider graphs in which for any induced subgraph, $\Delta \leq \omega-1+k$ holds, where $\Delta$ is the maximum degree and $\omega$ is the maximum clique number of the subgraph. We give a finite forbidden induced subgraph characterization for every $k$.

As an application, we find some results on the chromatic number $\chi$ of a graph. B. Reed stated the conjecture that for every graph, $\chi \leq\left\lceil\frac{\Delta+\omega+1}{2}\right\rceil$ holds. Since this inequality is fulfilled by graphs in which $\Delta \leq \omega+2$ holds, our results provide a hereditary graph class for which the conjecture holds.

Keywords: maximum clique, maximum degree, structural characterization of families of graphs, coloring of graphs.
\end{abstract}

\section{Introduction}

A graph class $\mathcal{G}$ is called hereditary if for every graph $G \in \mathcal{G}$, every induced subgraph of $G$ is also a member of $\mathcal{G}$. Hence, if we describe a graph class $\mathcal{G}$ by excluding a (not necessarily finite) set of graphs as induced subgraphs, then this graph class is hereditary.

A famous hereditary graph class is the class of perfect graphs. If $H$ is a perfect graph, then for $H$ and all its induced subgraphs the clique number and the chromatic number have the same value. By the Strong Perfect Graph Theorem [9], those graphs can be explicitely described by a set of forbidden induced subgraphs, the odd holes and the odd anti-holes. This class is of great interest from both a structural and an algorithmic point of view, see for example [11] and [12].

The advantage of such a structural description of a graph class is that it may lead to algorithmic results. For example, the maximum stable set problem, the

Oliver Schaudt

Université Pierre et Marie Curie, Combinatoire et Optimisation, 4 place Jussieu, 75252 Paris, France, E-mail: schaudto@uni-koeln.de

Vera Weil

Universität zu Köln, Institut für Informatik, Weyertal 80, 50931 Cologne, Germany E-mail: vweil@uni-koeln.de 
maximum clique problem, the coloring problem and the clique covering problem are solvable in polynomial time for perfect graphs [13].

Given the clique number $\omega$ and the maximum degree $\Delta$ of a graph, necessarily $\omega \leq \Delta+1$ holds. On the other hand, it is not possible to give a lower bound on $\omega$ in terms of $\Delta$ only: Since by Brook's Theorem [6], $\Delta \geq \chi-1$ holds, the Mycielski graphs $M_{p}, p \in \mathbb{N}$, a sequence of triangle-free graphs with chromatic number $p$ [14], yield an example for a family of graphs where the difference between $\omega$ and $\Delta$ can not be bounded by a constant.

If we require for a graph that the difference between $\Delta$ and $\omega$ is bounded, then this does not mean that the difference between the maximum degree and the clique number of the induced subgraphs is also bounded. For a given $p \in \mathbb{N}$ it is straightforward to construct a graph $H$ where the difference between $\omega(H)$ and $\Delta(H)$ equals 0 , but a graph $G$ is induced in $H$ where $\Delta(G)-\omega(G)>p$.

But what are the graphs that guarantee that for every induced subgraph of a host graph, the difference between the maximum degree and the clique number is at most as large as the difference between the maximum degree and the clique number of the host graph?

To reformulate and eventually answer this question, we define new hereditary graph classes: For a given number $k$, we require for every graph $H$ that the difference between the maximum degree and the clique number of every induced subgraph of $H$ is bounded by $k$. So for a given $k$, what are the graphs contained in the respective graph class? Or, from the original point of view: Given a graph $H$ where the difference between $\Delta$ and $\omega$ is bounded by a constant $k$, does $H$ belong to the respective hereditary graph class such that we can guarantee that the difference is bounded for every induced subgraph of $H$ ?

More precisely, we study the following graph classes. For every $k \in \mathbb{N}_{0}$, let $\Omega_{k}$ be the class of graphs $H$ for which $\Delta(G)+1 \leq \omega(G)+k$ holds for all induced subgraphs $G$ of $H$. Note that this includes $H$ itself. We answer the above questions in the following way: For every $k$, we describe all graphs contained in $\Omega_{k}$ by giving a minimal forbidden induced subgraph characterization. Moreover, we are able to prove that the order of the respective minimal forbidden induced subgraph set is finite. This gives that the problem of recognition of such graphs can be solved in polynomial time.

Finally, our results lead to a hereditary graph class for which Reed's Conjecture (Conjecture 1 stated below) holds. This conjecture is a highly non-trivial bound for the chromatic number in terms of the clique number and the maximum degree.

Conjecture 1 ([1]) For all graphs $H$ the following inequality holds:

$$
\chi(H) \leq\left\lceil\frac{\Delta(H)+\omega(H)+1}{2}\right\rceil .
$$

Note that in this work, we have to distinguish between induced subgraphs and (partial) subgraphs. Since we deal with graph invariants, we are allowed to treat isomorphic graphs as identical. For example, if a graph $G$ is an induced subgraph of a graph $H$ and $G$ is isomorphic to a graph $L$, then we say that $L$ is an induced subgraph of $H$.

Let $\Phi_{k}$ be the set of minimal forbidden induced subgraphs of $\Omega_{k}$. Hence for every graph $F \in \Phi_{k}, F \notin \Omega_{k}$ and all proper induced subgraphs of $F$ are contained in $\Omega_{k}$. Observe that $H \in \Omega_{k}$ if and only if $H$ is $\Phi_{k}$-free. In the following, we 
show how to iteratively construct the set $\Phi_{k}$ for each $k \in \mathbb{N}$, starting from $\Phi_{0}$. We explicitly draw $\Phi_{k}$ for all $k=1,2,3$.

\section{General results}

Our results are primarily based on Theorem 1, which characterizes the minimal forbidden induced subgraphs of $\Omega_{k}$ by three properties. We use the following notions: A vertex is dominating in a graph if it is adjacent to all other vertices of the graph. A clique is maximal in a graph if it is not contained in a clique of larger size. A maximal clique that is of largest size in a graph is called a maximum clique of the graph.

Theorem 1 Let $G$ be a graph. $G \in \Phi_{k}$ if and only if the following conditions hold:

1. G has a unique dominating vertex $v$,

2. the intersection of all maximum cliques of $G$ contains solely $v$,

3. $\Delta(G)=\omega(G)+k$.

In particular, $\Delta(G)=|V(G)|-1$ and $\omega(G)=|V(G)|-k-1$.

Proof Note that if the conditions 1, 2 and 3 hold for a graph $G$, then the dominating vertex $v$ is of maximum degree, thus $\Delta(G)=|V(G)|-1$. Hence, by Condition 3, $\Delta(G)=\omega(G)+k$, and therefore $\omega(G)=|V(G)|-k-1$.

Let $G \in \Phi_{k}$. We show that the three conditions hold. Note that since $G$ is minimal, all induced subgraphs of $G$ are contained in $\Omega_{k}$ except for $G$ itself. Thus $\Delta(G)>\omega(G)+k-1$.

Condition 1: Choose a vertex $v$ of maximum degree in $G$ and observe that the graph induced in $G$ by the vertex set $v \cup N(v)$, say $H$, is not in $\Omega_{k}$, since $\Delta(H)=\Delta(G)>\omega(G)+k-1 \geq \omega(H)+k-1$. Assume there exists a vertex $y \in V(G) \backslash N(v) \cup\{v\}$ and note that $\Delta(G-y)=\Delta(G)$ and that $\omega(G-y) \leq \omega(G)$. Hence, $\Delta(G-y)>\omega(G-y)+k-1$, contradicting the minimality of $G$. That is, $G \cong H$, and so $v$ is a dominating vertex of $G$.

Assume there exists $x \in V(G) \backslash\{v\}$ such that $\omega(G-x)=\omega(G)-1$. Then

$$
\Delta(G-x)=\Delta(G)-1 \geq \omega(G)+k-1=\omega(G-x)+k .
$$

This implies that $G-x \notin \Omega_{k}$, a contradiction to the minimality of $G$. Hence $v$ is the unique dominating vertex of $G$.

Condition 2: Like above, for every $x \in V(G) \backslash\{v\}$ it holds that $\omega(G-x)=\omega(G)$. In particular, the intersection of all maximum cliques of $G$ solely contains $v$.

Condition 3: Because of Condition 1, the degree of any $x \in V(G) \backslash\{v\}$ is at most $\Delta(G)-2$. Thus $\Delta(G-x)=\Delta(G)-1$ for any vertex $x \in V(G) \backslash\{v\}$. Like above, $\omega(G-x)=\omega(G)$ for any $x \in V(G) \backslash\{v\}$. Assume $\Delta(G) \geq \omega(G)+k+1$ and let $x \in V(G) \backslash\{v\}$. Then

$$
\Delta(G-x)=\Delta(G)-1 \geq(\omega(G)+k+1)-1=\omega(G-x)+k .
$$

That is, $G-x \notin \Omega_{k}$, a contradiction. Hence $\Delta(G)=\omega(G)+k$.

On the other hand, let $G$ be a graph having the properties of Condition 1, 2 and 3. Since $\Delta(G)=\omega(G)+k>\omega(G)+k-1, G$ is a forbidden induced subgraph for every graph contained in $\Omega_{k}$. 
To see that $G$ is minimal, assume the opposite. Let $L$ be a forbidden induced subgraph of minimal order that is an induced subgraph of $G$, hence $L \in \Phi_{k}$. By assumption, $G \neq L$. We already proved that $L$ has the following properties: $L$ has a unique dominating vertex $y$, the intersection of all maximum cliques of $L$ contains solely $y$ and $\Delta(L)=\omega(L)+k$. Since the graph induced by $(V(L) \backslash\{y\}) \cup\{v\}$ is isomorphic to $L$, we can assume that $v=y$. Let $S=V(G)-V(L)$ and let $|S|=s$. Then $\omega(L)+k=\Delta(L)=\Delta(G)-s=\omega(G)+k-s$, thus $S$ lies in the intersection of all maximum cliques of $G$. Hence $S=\emptyset$, therefore $G=L$, a contradiction. It follows that $G \in \Phi_{k}$.

Our next theorem, Theorem 2, provides a bound in terms of $k$ on the order of the minimal forbidden induced subgraphs of $\Omega_{k}$. For $s, t \in \mathbb{N}$, the graph $K_{s, t}$ is the complete bipartite graph where the blocks are of size $s$ and $t$, respectively.

Theorem 2 Let $G \in \Phi_{k}$. Then $k+2 \leq \Delta(G) \leq 2 k+2$ and $\Delta(G)=k+2$ if and only if $G \cong K_{1, k+2}$.

To shorten the proof of Theorem 2, we use Proposition 1, based on a lemma of Hajnal [2], the latter given here in the reformulation stated in [3]. To simplify notation, we write $\bigcup \mathcal{C}$ instead of $\bigcup_{\mathcal{A} \in \mathcal{C}} \mathcal{A}$. The same holds for $\cap \mathcal{C}$.

Lemma 1 ([3]) Let $G$ be a graph and let $\mathcal{C}$ be a collection of maximum cliques in $G$. Then $|\cap \mathcal{C}|+|\cup \mathcal{C}| \geq 2 \omega(G)$.

Proposition 1 is a straightforward consequence of Lemma 1.

Proposition 1 Let $G$ be a graph and let $2 \omega(G)>|V(G)|$. Then the intersection of all maximum cliques is not empty.

We want to emphazise that the statement of Proposition 1 is false if $2 \omega(H)=$ $|V(H)|$, consider for example the graph $S_{3}$ (cf. Figure 2). Furthermore, maximum cliques can not be replaced by maximal cliques of size at least $\frac{1}{2}|V(H)|+1$, even if all maximal cliques intersect pairwise. Consider for example the graph of order $3 t+3, t \geq 2$, sketched in Figure 2. Gray areas between two black lines represent the set of all edges between the sets adjacent to that area. The graph contains four maximal cliques, three of which are of size $2 t+1>\frac{3 t+3}{2}$, while the only maximal clique that is maximum is of size $3 t$. All maximal cliques intersect pairwise, but the intersection of all maximal cliques is empty.

Proof (Proof of Theorem 2) Let $G \in \Phi_{k}$. We have to show that $k+2 \leq \Delta(G) \leq$ $2 k+2$ and $\Delta(G)=k+2$ if and only if $G \cong K_{1, k+2}$. For this, let $v$ be the unique dominating vertex of $G$. Note that $\omega(G-v)=\omega(G)-1$ and that, by Theorem 1, the intersection of all maximum cliques of $G-v$ is empty. According to Proposition $1,2 \omega(G-v) \leq \Delta(G)=|V(G-v)|$. Hence,

$$
\begin{aligned}
& 2 \omega(G)-2 \leq \Delta(G) \\
& \Rightarrow \quad \omega(G) \leq \Delta(G)-\omega(G)+2 \\
& \Rightarrow \quad \omega(G) \leq k+2 \text {. }
\end{aligned}
$$

Therefore $\Delta(G)=\omega(G)+k \leq 2 k+2$. It is clear that $\omega(G) \geq 2$, thus $\Delta(G)=$ $\omega(G)+k \geq k+2$. Finally, $\Delta(G)=k+2$ if and only if $\omega(G)=2$ if and only if $G \cong K_{1, k+2}$. 
The next theorem, Theorem 3, shows how to list all graphs contained in $\Phi_{k+1}$, given $\Phi_{k}$.

Theorem 3 Any graph $H \in \Phi_{k+1}$ is constructed by adding one or two vertices and some incident edges to a graph $G \in \Phi_{k}$ such that

- all new vertices are adjacent to the dominating vertex $v$ of $G$,

- if only one vertex is added, then $\omega(H)=\omega(G)$,

- if two vertices are added, then the intersection of the maximum cliques contains solely $v$ and $\omega(H)=\omega(G)+1$.

Vice versa, any graph constructed this way belongs to $\Phi_{k+1}$.

To shorten and clarify the proof of Theorem 3, we use Proposition 2. To concentrate on the main arguments in the proof of Proposition 2, we first state and prove the technical Lemma 2 and Lemma 3.

Lemma 2 Let $G$ be a graph and let $\mathcal{C}$ be a set of cliques in $G$ with non-empty intersection $A$. If $B$ is a maximal clique in $G$ such that $A \nsubseteq B$, then $B \nsubseteq \cup \mathcal{C}$. In particular, $V(G) \neq \bigcup \mathcal{C}$.

Proof Let $G, \mathcal{C}, A$ and $B$ be as described in Lemma 2. Then there exists at least one vertex $a \in A \backslash B$. Assume $B \subseteq \cup \mathcal{C}$. Since every vertex in $\bigcup \mathcal{C}$ is adjacent to every vertex in $A$, the set $B \cup\{a\}$ is a clique of larger size than $B$, contradicting the maximality of $B$.

Lemma 3 Let $G$ be a graph such that the intersection of all maximum cliques in $G$ is empty and let $\omega(G) \geq 2$. Furthermore, let $x, y \in V(G)$ be such that every maximum clique contains $x$ or $y$, but not both. Then the intersection of all maximum cliques of $G-\{x, y\}$ is empty.

Proof Let $G, x$ and $y$ be as described in Lemma 3. Note that $\omega(G-\{x, y\})=$ $\omega(G)-1$. In particular, if $C$ is a maximum clique of $G$, then $C \backslash\{x, y\}$ is a maximum clique of $G-\{x, y\}$. Hence the intersection of all maximum cliques of $G$ contains the intersection of all maximum cliques of $G-\{x, y\}$, which therefore is empty.

Proposition 2 observes that any graph whose maximum cliques have an empty intersection has a proper induced subgraph where again, the maximum cliques have an empty intersection.

Proposition 2 Let $G$ be a graph such that the intersection of all maximum cliques is empty. Then there exists a nonempty set $S \subseteq V(G)$ of at most two vertices such that the intersection of all maximum cliques of $G-S$ is empty. If $|S|=1$, then $\omega(G-S)=\omega(G)$, if $|S|=2$, then $\omega(G-S)=\omega(G)-1$.

Proof Let $G$ be a graph such that the intersection of all maximum cliques of $G$ is empty. If for some $x \in V(G)$ the intersection of all maximum cliques of $G-x$ is empty, then choose $S=\{x\}$ and the claim of Proposition 2 follows. Hence, we consider the case in which for every vertex $v \in V(G)$, the intersection of all maximum cliques of $G-v$ is not empty. In particular, this implies that every vertex is contained in at least one maximum clique.

Let $\mathcal{C}$ be an inclusionwise maximal set of maximum cliques in $G$ such that the intersection $A$ of these cliques is not empty. Hence any maximum clique not in 
$\mathcal{C}$ does not intersect with $A$. Since the intersection of all maximum cliques of $G$ is empty, there exists a maximum clique $L \notin \mathcal{C}$. By Lemma $2, L \backslash \bigcup \mathcal{C} \neq \emptyset$. Let $y \in V(G) \backslash \cup \mathcal{C}$. Assume there is a maximum clique not in $\mathcal{C}$ that does not contain $y$. Then the maximum cliques of $G-y$ do not intersect, a contradiction.

Hence $y$ is a member of every maximum clique that does not contain $A$, and vice versa, since any maximum clique that contains $A$ is in $\mathcal{C}$. Choose $x \in A$ and observe that $\omega(G-\{x, y\})=\omega(G)-1$. By Lemma 3, the intersection of all maximum cliques of $G-\{x, y\}$ is empty.

We are now in the position to prove Theorem 3.

Proof (Proof of Theorem 3) Given the set $\Phi_{k}$, we construct the set $\Phi_{k+1}$ in the following way. For every $G \in \Phi_{k}$, we construct two sets of graphs contained in $\Phi_{k+1}$ : The set $S_{1}(G)$ consists of graphs with $|V(G)|+1$ vertices and the set $S_{2}(G)$ consists of graphs with $|V(G)|+2$ vertices. By Theorem 1, $G$ has a unique dominating vertex, say $v$. To construct $S_{1}(G)$, add a new neighbor $a$ to $v$ and call that graph $G_{a}$.

Note that a supergraph of a graph may have additional edges and vertices. Add all graphs $\tilde{G}$ to $S_{1}(G)$ that are supergraphs of $G_{a}$ with the additional conditions that $\tilde{G}-a \cong G$ and that $a$ is not contained in all maximum cliques of $\tilde{G}$.

To construct $S_{2}(G)$, add two new neighbors $b, c$ to $v$ that are not adjacent to each other and call that graph $G_{b, c}$. Add all graphs $\tilde{G}$ to $S_{2}(G)$ that are supergraphs of $G_{b, c}$ with the additional conditions that $\tilde{G}-\{b, c\} \cong G, \omega(\tilde{G})=\omega(G)+1$ and the intersection of all maximum cliques contains solely $v$. See Figure 1 for an illustration of the transition from $\Phi_{0}$ to $\Phi_{1}$.

We claim that the union of $S_{1}(G)$ and $S_{2}(G)$ of all $G \in \Phi_{k}$ is exactly $\Phi_{k+1}$, that is,

$$
\bigcup_{G \in \Phi_{k}}\left(S_{1}(G) \cup S_{2}(G)\right)=\Phi_{k+1}
$$

To prove our claim, let $H$ be a graph constructed from a graph $G \in \Phi_{k}$ by the steps described above. We show that $H \in \Phi_{k+1}$. By Theorem 1 , we have to check the following three conditions:

1. $H$ has a unique dominating vertex $v^{\prime}$,

2. the intersection of all maximum cliques of $H$ contains solely $v^{\prime}$,

3. $\Delta(H)=\omega(H)+k+1$.

The unique dominating vertex of $G$ is also the unique dominating vertex of $H$, hence Condition 1 is fulfilled.

We first assume $H \in S_{1}(G)$. Hence, let $s$ be the vertex added to $V(G)$ and observe that $\Delta(H)=\Delta(G)+1$. Furthermore, $\omega(H)=\omega(G)$, otherwise all cliques of size $\omega(G)+1$ would contain $s$, a contradiction. Hence, all maximum cliques of $G$ are also maximum cliques of $H$. So, the intersection of all maximum cliques of $H$ contains solely $v$. This proves Condition 2 .

Finally, $\Delta(H)=\Delta(G)+1=\omega(G)+k+1=\omega(H)+k+1$, that is, condition 3 holds. Thus, by Theorem $1, H \in \Phi_{k+1}$.

Assume $H \in S_{2}(G)$. The intersection of all maximum cliques contains solely the unique dominating vertex of $H$, that is, Condition 2 holds. Observe that $\Delta(H)=$ $\Delta(G)+2$ and that $\omega(H)=\omega(G)+1$. Condition 3 holds since $\Delta(H)=\Delta(G)+2=$ $\omega(G)+k+2=\omega(H)+k+1$. Hence, by Theorem $1, H \in \Phi_{k+1}$. 
Conversely, let $H \in \Phi_{k+1}$. Let $v$ be the unique dominating vertex of $H$ and let $G=H-v$. Then, by Proposition 2 , there exists a nonempty set $S \subseteq G$ such that $1 \leq$ $|S| \leq 2$ and the intersection of all maximum cliques of $G-S$ is empty In particular, $G-S$ does not contain a dominating vertex, hence $v$ is the unique dominating vertex in $H-S$. Note that $\Delta(H-S)=\Delta(H)-|S|$. Again, by Proposition 2 , if $|S|=1$, then $\omega(G-S)=\omega(G)$. Therefore $\omega(H-S)=\omega(H)=\Delta(H)-1-k=\Delta(H-S)-k$. By Theorem $1, H-S \in \Phi_{k}$ and $H \in S_{1}(H-S)$. If $|S|=2$, then $\omega(G-S)=\omega(G)-1$, hence $\omega(H-S)=\omega(H)-1=\Delta(H)-2-k=\Delta(H-S)-2$. By Theorem 1, $H-S \in \Phi_{k}$ and $H \in S_{2}(H-S)$.

Theorem 2 and Theorem 3 have an important consequence in common: On the one hand, Theorem 2 gives a bound for the order of minimal forbidden induced subgraphs, mainly based on the structural result of Proposition 1 . Hence, for any fixed $k, \Phi_{k}$ is a subset of the set of graphs that have at most $2 k+3$ vertices, and therefore is finite. Hence, the characterization given in Theorem 1 leads to the result that the set of minimal forbidden induced subgraphs is finite for any given $k \in \mathbb{N}_{k}$.

On the other hand, Theorem 3 and its proof provide a construction manual that leads to the set $\Phi_{k+1}$ by adding, roughly speaking, at most two vertices and different edge sets to every graph contained in the set $\Phi_{k}$. Thus, if $\Phi_{k}$ is finite, then so is $\Phi_{k+1}$. Therefore, in order to show that $\Phi_{k}$ is finite for any given $k \in \mathbb{N}_{0}$, it suffices to show that $\Phi_{0}$ is finite, what is the case as stated in Theorem 4 . In particular, by Theorem 3 it is possible to construct the sets $\Phi_{k}$ for all $k \in \mathbb{N}$, given $\Phi_{0}$. We explicitely draw $\Phi_{0}$ in Theorem 4 .

For $s \in \mathbb{N}, P_{s}$ denotes the path on $s$ vertices.

Theorem $4 \Phi_{0}=\left\{P_{3}\right\}$. That is, $\Omega_{0}$ consists of unions of complete graphs.

Proof According to Theorem 1 and Theorem 2, $\Phi_{0}$ contains all graphs $G$ such that $2 \leq \Delta(G)=\omega(G) \leq 2, G$ has a dominating vertex $v$ and the intersection of all maximum cliques of $G$ contains only $v$. But then, $G \cong P_{3}$.

As mentioned above, as a consequence of Theorem 2 or as a consequence of Theorem 3 together with Theorem 4, we state the following observation:

Observation 1 For any given $k \in \mathbb{N}_{0}, \Phi_{k}$ is finite.

In the following, we explicitly draw the sets $\Phi_{1}$ and $\Phi_{2}$. Note that Theorem 5 is a consequence of Theorem 3. However, we give a direct proof that spares the reader to compute the sets $S_{1}\left(P_{3}\right)$ and $S_{2}\left(P_{3}\right)$ mentioned in the proof of Theorem 3. By $\alpha(H)$ we denote the maximum size of a stable set contained in a graph $H$. For the graphs in the set $\Phi_{1}$, cf. Figure 1 . Note that the butterfly graph is also known as bowtie or hourglass graph.

Theorem $5 \Phi_{1}=\left\{\right.$ claw, gem, $W_{4}$, butterfly $\}$.

Proof Let $H \in \Phi_{1}$. According to Theorem $2, \Delta(H)=3$ if and only if $H \cong K_{1,3}$. If $\Delta(H)=4$, let $v$ be the unique dominating vertex of $H$ and consider $G=H-v$. Note that if $\alpha(G)=1$, then $H$ is a clique, what is impossible since $v$ is the unique dominating vertex. If $\alpha(G) \geq 3$, then $H$ contains a claw as induced subgraph, contradicting the minimality of $G$, since the claw is a minimal forbidden induced subgraph. 

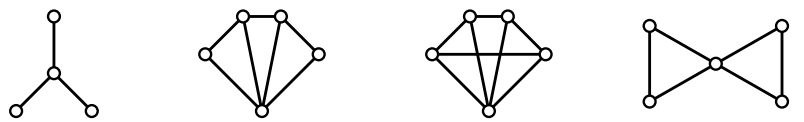

Fig. 1 The graphs claw, gem, $W_{4}$, butterfly. In the proof of Theorem $3,\{$ claw $\}=S_{1}\left(P_{3}\right)$, $\left\{\right.$ gem, $W_{4}$, butterfly $\}=S_{2}\left(P_{3}\right)$.

Hence $G$ is isomorphic to a bipartite graph consisting of 4 vertices and $\alpha(G)=$ 2 , which implies that every block of the bipartition consists of exactly two vertices and $G$ contains at least two vertex disjoint edges. Then $G \cong K_{2} \cup K_{2}$, hence $H \cong$ butterfly, or $G \cong P_{4}$, hence $H \cong$ gem, or $G \cong C_{4}$, hence $H \cong W_{4}$.

Note that the graphs in $\Omega_{1}$ are in particular (claw, gem, $W_{4}$ )-free. This is the minimal forbidden induced subgraph characterization of line graphs of multigraphs without triangles [4], called dominoes [5]. Hence $\Omega_{1}$ is the class of butterfly-free dominoes.

The set $\Phi_{2}$ described below in Theorem 6 can be found by using Theorem 3 and Theorem 5 . However, there is a shorter way to prove Theorem 6 using Lemma 4 and Proposition 3. By $K_{n}, n \in \mathbb{N}$, we denote the complete graph on $n$ vertices. A $K_{2 s}-s e$ is the graph $K_{2 s}$ where a perfect matching is removed. For example, $K_{4}-2 e \cong C_{4}$.

Lemma 4 Let $G$ be a graph with $2 s$ vertices, $s \in \mathbb{N}_{0}$, such that $K_{s} \cup K_{s}$ is a subgraph of $G$. Then $\omega(G)=s$ if and only if $G$ is a subgraph of $K_{2 s}-$ se.

Proof Let $G$ be a supergraph of $K_{s} \cup K_{s}$ on $2 s$ vertices. Then $\omega(G) \geq s$ and $|V(G)|=2 s$, hence $G \subseteq K_{2 s}$. Let $A_{1} \cup A_{2}$ be a partition of $V(G)$ such that $A_{1}, A_{2} \cong K_{s}$. Consider the complement $\bar{G}$ of $G$, a bipartite graph with stable sets $B_{1}$ and $B_{2}$ corresponding to the cliques $A_{1}$ and $A_{2}$ of $G$. In particular, $\left|B_{1}\right|=\left|B_{2}\right|$.

According to Hall's Theorem [10], $\bar{G}$ has a perfect matching if and only if for every set $X \subseteq B_{1},|X| \leq\left|N_{\bar{G}}(X)\right|$, where $N_{\bar{G}}(X)$ is the union of neighbors of $x \in X$ in $\bar{G}$. Thus $G$ is not a subgraph of $K_{2 s}-$ se if and only if in $B_{1}$ there is a set $X$ such that $|X|>\left|N_{\bar{G}}(X)\right|$. Note that there is no edge between $X$ and $B_{2} \backslash N_{\bar{G}}(X)$ in $\bar{G}$, hence in $G, X \cup\left(B_{2} \backslash N_{\bar{G}}(X)\right)$ forms a clique. Hence, in $B_{1}$, there is a set $X$ such that $|X|>\left|N_{\bar{G}}(X)\right|$ if and only if in $G$ there is a clique of size $|X|+\left|B_{2} \backslash N_{\bar{G}}(X)\right|=|X|+s-\left|N_{\bar{G}}(X)\right| \geq s+1$ if and only if $\omega(G) \geq s+1$.

Note that the statement in the following Proposition 3 is false if we only require that the maximum cliques intersect in $\omega(G)-2$ vertices, consider for example the graph $S_{3}$ (cf. Figure 2) in which $\omega\left(S_{3}\right)=3$ and every two maximum cliques intersect in one vertex.

Proposition 3 Let $G$ be a graph with $\omega(G) \geq 2$ such that every two maximum cliques intersect in $\omega(G)-1$ vertices. Then the intersection of all maximum cliques is not empty.

Proof Let $G$ be a graph with $\omega(G) \geq 2$ such that every two maximum cliques intersect in $\omega(G)-1$ vertices but the intersection of all maximum cliques is empty. Let $\mathcal{C}$ be an inclusionwise maximal set of maximum cliques such that the intersection 


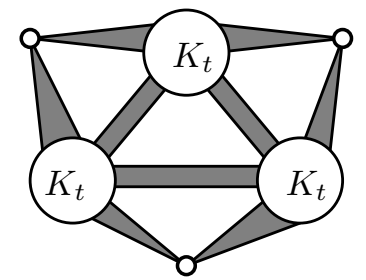

Fig. 2 Generalized $S_{3}$. Gray areas represent all edges between the vertex sets adjacent to that area. The sketched graph is isomorphic to the $S_{3}$ if $t=1$.

$A$ of these cliques is not empty. Since the intersection of all maximum cliques is empty, there exists a maximum clique $D \notin \mathcal{C}$ such that $D \cap A=\emptyset$. Since each two maximum cliques intersect in $\omega(G)-1$ vertices, $|A|=1$ and for all $B \in \mathcal{C}$, $D \cap B=B-A$. Note that if $|D \cap \bigcup \mathcal{C}| \geq \omega(G)$, then $(D \cap \bigcup \mathcal{C}) \cup A$ forms a clique of size $\omega(G)+1$, a contradiction.

Hence $\omega(G)-1 \geq|D \cap \bigcup \mathcal{C}|=|\bigcup \mathcal{C}-A| \geq \omega(G)-1$. It follows that $\mathcal{C}$ consists of one clique, hence $|A|=\omega(G)$, a contradiction.

Having proved those auxiliary results, we can finally state Theorem 6 . For the graph $S_{3}$, which is sometimes called the Hajós graph, cf. Figure 2.

Theorem 6 Let $G$ be a graph. $G \in \Phi_{2}$ if and only if $G$ contains a dominating vertex $v$ and one of the following holds:

1. $G-v$ is isomorphic to $S_{3}$ or the complement of $K_{4}$.

2. $G-v$ is a $K_{3}$-free supergraph of $K_{2} \cup K_{2} \cup K_{1}$ on 5 vertices.

3. $G-v$ is a supergraph of $K_{3} \cup K_{3}$ and a subgraph of $K_{6}-3 e$.

Proof Let $G$ be a graph such that $G$ has a dominating vertex $v$ and $G-v$ has Property 1, 2 or 3 formulated in Theorem 6 . Then vertex $v$ is uniquely dominating in $G$, the intersection of all maximum cliques of $G$ solely contains $v$ and $\Delta(G)=$ $\omega(G)+2$. According to Theorem $1, G \in \Phi_{2}$.

On the other hand, let $G \in \Phi_{2}$, let $v$ be the unique dominating vertex of $G$ and let $H=G-v$. According to Theorem $2,4 \leq|V(H)| \leq 6$ and $|V(H)|=4$ if and only if $H \cong K_{1,4}$. In this case, $H$ has Property 1 .

If $|V(H)|=5$, then $\omega(H)=2$. By minimality, $\alpha(H) \leq 3$, hence $H$ is a $K_{3}$-free supergraph on 5 vertices of the $K_{2} \cup K_{2} \cup K_{1}$, thus has Property 2 .

If $|V(H)|=6$, then $\omega(H)=3$. If furthermore $H$ contains two vertex disjoint maximum cliques of size 3 , then according to Lemma $4, K_{3} \cup K_{3} \subseteq H \subseteq K_{6}-3 e$. Hence $H$ has Property 3.

If every two maximum cliques of $H$ intersect in at least two vertices, then, according to Proposition 3, the intersection of all maximum cliques of $H$ is not empty, hence, by Theorem $1, G \notin \Phi_{2}$, a contradiction.

Finally, we consider the case in which all maximum cliques intersect pairwise and there are two maximum cliques $A, B$ that intersect in exactly one vertex $y$. Then there exists $x \notin A \cup B$ such that $x$ is not adjacent to $y$, otherwise $y$ is another dominating vertex, a contradiction to Theorem 1 . Since the intersection of all 

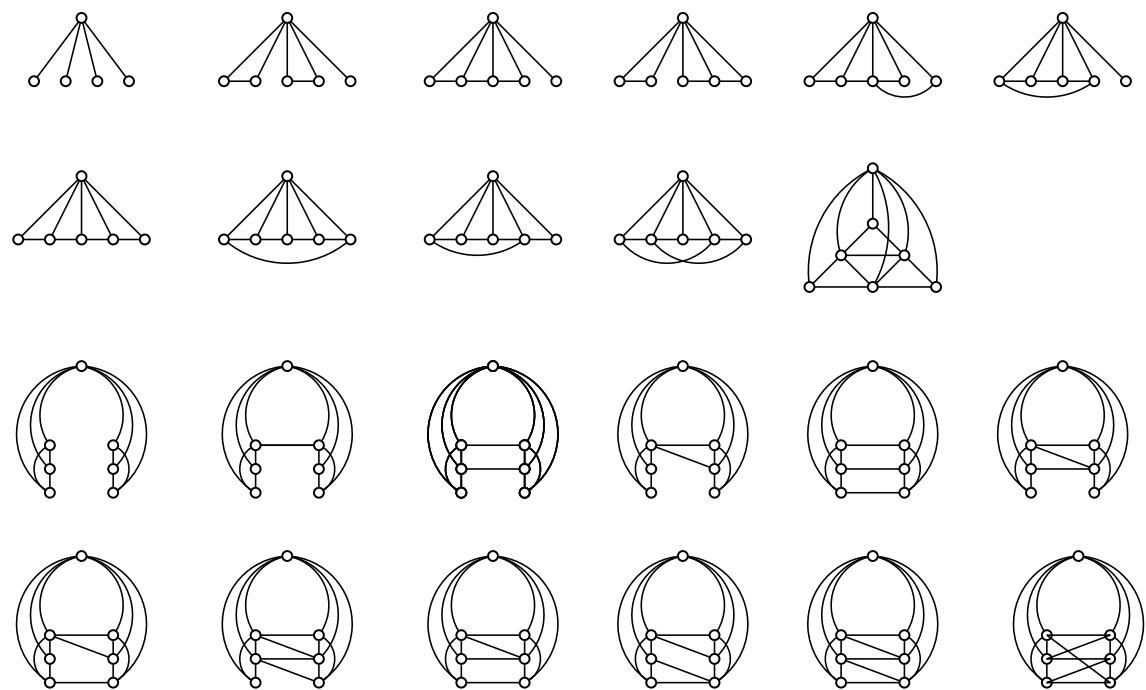

Fig. 3 The set $\Phi_{2}$, that is, the minimal forbidden induced subgraphs of $\Omega_{2}$.

maximum cliques is empty, there exists at least one maximum clique $C$ such that $y \notin C$.

Let $b \in B \cap C$. If $|C \cap A|=2$, then $(A \cup B) \cap C$ together with $y$ forms a clique of size 4 , a contradiction. It follows that $H$ is a supergraph of $S_{3}$. Note that any proper graph on six vertices that is a supergraph of $S_{3}$ either contains two vertex disjoint maximum cliques, a case we handled before, or contains a $K_{4}$, contradicting $\omega(H)=3$. Thus $H \cong S_{3}$, and $H$ has Property 1 .

All graphs contained in $\Phi_{2}$ are shown in Figure 3.

\section{An application: Results on the chromatic number}

Recall that the chromatic number $\chi$ of a graph is bounded from below by the maximum clique number and from above by $\Delta+1$ by Brooks' Theorem [6]. We restate this theorem omitting the condition of connectedness:

Theorem 7 (Brooks [6]) Let $G$ be a graph. Then $\chi(G) \leq \Delta(G)+1$. If $\chi(G)=$ $\Delta(G)+1$, then $G$ has a connected component that is an odd cycle or a complete graph.

Hence for a given graph, $\chi$ is between $\omega$ and $\Delta+1$. From this point of view, for each $k$, the chromatic number of $\Omega_{k}$ is limited to at most $k+1$ different values. In [1], Reed conjectured the following:

Conjecture 1 For all graphs $G$ the following inequality holds:

$$
\chi(G) \leq\left\lceil\frac{\Delta(G)+\omega(G)+1}{2}\right\rceil .
$$


For recent developments on this conjecture, the reader is refered to [7].

Note that $\Omega_{k} \subseteq \Omega_{k+1}$ for all $k$. Hence, if Reed's conjecture holds for $\Omega_{k+1}$, then it holds for $\Omega_{k}$.

Theorem 8 Let $G \in \Omega_{k}$. For all induced subgraphs $H$ of $G$,

$$
\left\lfloor\frac{\Delta(H)+\omega(H)+2-k}{2}\right\rfloor \leq \chi(H) .
$$

Moreover, for $k \leq 3$, for all induced subgraphs $H$ of $G$,

$$
\chi(H) \leq\left\lceil\frac{\Delta(H)+\omega(H)+1}{2}\right\rceil .
$$

Proof Let $G \in \Omega_{k}$ and let $H$ be an induced subgraph of $G$. Recall that $\Delta(H)+1-$ $k \leq \omega(H)$, hence

$$
\left\lfloor\frac{\Delta(H)+\omega(H)+2-k}{2}\right\rfloor \leq\left\lfloor\frac{2 \omega(H)+1}{2}\right\rfloor \leq \omega(H) \leq \chi(H) .
$$

In particular, if $k \leq 3$, then $\Delta(H) \leq \omega(H)+2$. If $H$ is an induced subgraph such that $\Delta(H)=\chi(H)+1$, then (2) obviously holds. For all other induced subgraphs $H, \chi(H) \leq \Delta(H)$, thus (2) holds by

$$
\chi(H) \leq \Delta(H)=\left\lceil\frac{2 \Delta(H)-1}{2}\right\rceil \leq\left\lceil\frac{\Delta(H)+\omega(H)+1}{2}\right\rceil .
$$

Hence, Theorem 8 shows that Conjecture 1 holds for $k \leq 3$. The reader is refered to [8] for further graph classes in which Reed's Conjecture holds.

We call a graph Reed-perfect if for all induced subgraphs $H$ of $G$,

$$
\left\lfloor\frac{\Delta(H)+\omega(H)+1}{2}\right\rfloor \leq \chi(H) \leq\left\lceil\frac{\Delta(H)+\omega(H)+1}{2}\right\rceil .
$$

Informally speaking, the Reed-perfect graphs are the graphs for which $\chi$ meets the bound provided by Conjecture 1 . We find a short characterization of the sets $\Omega_{0}$ and $\Omega_{1}$ in terms of the bound of Conjecture 1:

Theorem 9 Let $G$ be a graph. Then the following holds:

1. $G \in \Omega_{0}$ if and only if for all induced subgraphs $H$ of $G, \chi(H)=\frac{\Delta(H)+\omega(H)+1}{2}$.

2. $G \in \Omega_{1}$ if and only if $G$ is Reed-perfect.

Proof To show the first claim, observe that by Theorem $4, \Omega_{0}$ consists of all unions of complete graphs. Hence for $G \in \Omega_{0}$,

$$
\chi(G)=\Delta(G)+1=\omega(G)=\frac{\Delta(G)+1+\omega(G)}{2} .
$$

On the other hand, if for all induced subgraphs the fraction given in the first claim is fulfilled, then the graphs are $P_{3}$-free, since

$$
2=\chi\left(P_{3}\right) \neq \frac{\Delta\left(P_{3}\right)+\omega\left(P_{3}\right)+1}{2}=\frac{5}{2} .
$$

These are exactly the unions of complete graphs.

To show the second claim, recall that if $G \in \Omega_{1}$, then by Theorem $8, G$ is Reed-perfect. On the other hand, let $G$ be a Reed-perfect graph. Hence $G$ is \{claw, $W_{4}$, gem, butterfly $\}$-free, since all of these graphs violate (3). 
We want to remark that the computation of $\chi$, given a Reed-perfect graph $G$, can be done in polynomial time. Note that if $G$ is not connected, it suffices to examine each compontent separately, hence we may assume that $G$ is connected. Recall that $G$ is the line graph of a multigraph without triangles, what implies that the number of maximal cliques in $G$ is linear. Hence, the computation of $\omega(G)$ can be done in polynomial time. Thus, if the parity of $\omega(G)$ and $\Delta(G)$ is not the same, then the value of $\chi$ follows from (3). In the other case, recall that $\Delta(G) \leq \omega(G) \leq \Delta(G)+1$, hence $\omega(G)=\Delta(G)$. Therefore, following (3), $\Delta(G) \leq \chi(G) \leq \Delta(G)+1$. By Brooks' Theorem [6], we simply check wether $G$ is an odd cycle or a complete graph. If this is not the case, $\chi(G)=\Delta(G)$.

Recall that a graph is called perfect if $\omega=\chi$ holds for all its induced subgraphs. A hole is a chordless cycle with at least 4 vertices. An antihole is the complement of a hole. An odd (anti-)hole is an (anti-)hole with an odd number of vertices. By the Strong Perfect Graph Theorem [9], a graph is perfect if and only if it is odd hole and odd antihole free.

To prove Theorem 10, we consider a hole $C_{n}, n \geq 7$, in which the vertices $v_{1}, \ldots, v_{n}$ are ordered clockwise. In the complement graph of $C_{n}$, the vertices $v_{2}, v_{4}, v_{5}, v_{6}, v_{7}$ induce a gem, hence we state the following observation:

Observation 2 Any antihole with at least 7 vertices contains a gem as induced subgraph.

The graphs that are both perfect and Reed-perfect are described in the following theorem:

Theorem 10 Let $G$ be a graph. $G$ is Reed-perfect and perfect if and only if for all its induced subgraphs $H$,

$$
\chi(H)=\left\lfloor\frac{\Delta(H)+\omega(H)+1}{2}\right\rfloor .
$$

Proof On the one hand, let $G$ be a perfect Reed-perfect graph. Then for each induced subgraph $H$ of $G, \chi(H)=\omega(H)$. Furthermore, by Theorem $9, \Delta(H)=$ $\omega(H)$ or $\Delta(H)+1=\omega(H)$. In both cases,

$$
\left\lfloor\frac{\Delta(H)+\omega(H)+1}{2}\right\rfloor=\omega(H)=\chi(H) .
$$

On the other hand, let $G$ be a graph that obeys to (4). Obviously, $G$ is Reedperfect, hence, by Theorem $5, G$ is gem-free. This implies, by Observation 2, that $G$ does not contain antiholes with at least 7 vertices. Further, for any odd hole $C$, $\chi(C)=3, \omega(C)=2$ and $\Delta(C)=2$ hold, hence $C$ does not obey to (4). It follows that $G$ is odd hole and odd antihole free, hence $G$ is perfect.

Note that the graphs described in Theorem 10 are in particular $\left(W_{4}\right.$, claw, gem, odd-hole)-free, hence are line graphs of bipartite multigraphs [4].

\section{Final remarks}

In this work we introduced a sequence of new graph families. A member of such a family has the property that for some fixed $k \in \mathbb{N}_{0}$, the graph and all its induced 
subgraphs comply with $\Delta+1 \leq \omega+k$. We showed that those graphs can be characterized by a finite set of minimal forbidden subgraphs. Moreover, we derived such a set for $k+1$ from the set computed for $k$. For $k=0,1$ and 2 , we drew the set of minimal forbidden subgraphs.

A further family of graphs, strongly related to a conjecture of Reed, was introduced, the so called Reed-perfect graphs. We proved that Reed-perfect graphs conincide with $\Omega_{1}$. Finally, we found a short description of those graphs that are Reed-perfect and perfect.

A future direction might include more related parameters. For example, we currently try to adapt our methods to the family of graphs for which the graph and all its induced subgraphs comply to $\Delta+1 \leq \chi+k$, for all $k \in \mathbb{N}_{0}$. Moreover, there exists recent literature on local variations of Reed's conjecture, including further conjectures that might fit to our family of graphs, see for example [15]. Also, extensions of Brooks' Theorem, as presented for example in [16], might lead to further results in combination with hereditary graph classes like those presented in this work.

\section{Acknowledgment}

We want to thank the reviewers for their many helpful comments.

\section{References}

1. B. Reed, OMEGA, DELTA, and CHI, J. Graph Theory 27 (1998), 177-212.

2. A. Hajnal, A theorem on k-saturated graphs, Canadian J. Math., 17:720-724, 1965.

3. D. Christofides, K. Edwards and A.D. King, A note on hitting maximum and maximal cliques with a stable set, Journal of Graph Theory, 73 (2013), 327-341.

4. Y. Metelsky, R. Tyshkevich, Line graphs of Helly hypergraphs, SIAM J. Discrete Math. 16, No.3, (2003), 438-448

5. T. Kloks, D. Kratsch and G. Müller, Dominoes, Lecture Notes in Comput. Sci. 903 (1995), 106-120.

6. R.L. Brooks, On colouring the nodes of a network, Math. Proc. Cambridge Philos. Soc. 37 (1941), 194-197.

7. A. King, Claw-free graphs and two conjectures on omega, Delta, and chi, Ph.D. dissertation, School of Computer Science, McGill University, Montreal, Canada, 2009.

8. N.R. Aravind, T. Karthick and C.R. Subramanian, Bounding $\chi$ in terms of $\omega$ and $\Delta$ for some classes of graphs, Discrete Math. 311 (2011), 911-920.

9. M. Chudnovsky, N. Robertson, P.D. Seymour and R. Thomas, The strong perfect graph theorem, Ann. Math. 164 (2006), 51-229.

10. P. Hall, On representatives of subsets, J. Lond. Math. Soc. 10 (1), 26-30.

11. B. Toft, Coloring, stable sets and perfect graphs, in R. Graham, M. Grötschel, L. Lovász, eds., Handbook of Combinatorics, Vol. I, North-Holland, 1995, 233 - 288.

12. M.C. Golumbic, Algorithmic Graph Theory and Perfect Graphs, Academic Press, New York 1980.

13. M. Grötschel, L. Lovász, A. Schrijver, Polynomial Algorithms for Perfect Graphs, NorthHolland Mathematics Studies - Topics on Perfect Graphs, Vol. 88 (1984), 325-356.

14. J. Mycielski, Sur les coloriages des graphes, Colloq. Math. 3 (1955), 161-162.

15. M. Chudnovsky, A. D. King, M. Plumettaz, and P. Seymour, A local strengthening of Reed's $\omega, \Delta, \chi$ conjecture for quasi-line graphs, SIAM J. Discrete Math. 27, No. 1 (2013), 95-108.

16. B. Farzad, M. Molloy, B. Reed, $(\Delta-k)$-critical graphs, Journal of Combinatorial Theory, Series B 93 (2005) $173-185$. 